\section{Fontes de inadequação das \\ recomendações internacionais \\ sobre requerimentos humanos de \\ energia para a população \\ brasileira*}

\section{Sources of inadequacy of the international recommendations on human energy requirements for the Brazilian population}

Mauricio Teixeira Leite de Vasconcellos

Diretoria de Pesquisas / Departamento de Metodologia

Fundação Instituto Brasileiro de Geografia e Estatística - IBGE

Av. República do Chile, $500-10^{\circ}$ andar

20031-170 - Rio de Janeiro, RJ - Brasil

mtlv@ibge.gov.br

* Apresentado como Painel no V Congresso Brasileiro de Epidemiologia. Curitiba, PR, Brasil. 23-27 de março de 2002.

\section{Resumo}

As recomendações internacionais sobre requerimentos humanos de energia (RHE), propostas pela FAO/WHO/UNU em 1985, representaram importante avanço metodológico, na medida em que os requerimentos passaram a ser baseados nos componentes de gasto energético do organismo, substituindo-se o método derivado da observação da ingestão de pessoas saudáveis. Esta modificação foi baseada na constatação de que a determinação dos RHE com base em ingestões observadas era um argumento circular, uma vez que estas não eram, forçosamente, aquelas que mantinham a massa corporal desejável e os níveis ótimos de atividade física para a saúde a longo prazo.

A reunião de 1994 do International Dietary Energy Consultative Group, publicada em 1996, bem como artigos de autores nacionais e estrangeiros, indicam que os principais problemas no método proposto estão relacionados:

- às equações de predição da taxa metabólica basal (TMB);

- aos valores de nível de atividade física (NAF);

- aos mecanismos de adaptação;

- ao método de cálculo dos requerimentos para menores de 10 anos de idade.

No caso brasileiro, as equações de predição tendem a superestimar a TMB de adultos (maiores de 18 anos) e os valores de NAF não são adequados à população, como indicam estudos recentes de medição da TMB e de estimação dos valores de NAF.

Comparando os RHE calculados pelo método fatorial (baseado na duração das atividades e nos correspondentes índices energéticos integrados) com aqueles obtidos pelo método simplificado (baseado nos valores recomendados de NAF), este artigo mostra que os valores recomendados de NAF:

- para as mulheres de 18 a 59 anos, determinam RHE de $11 \%$ a $17 \%$ acima do necessário para o grupo, podendo conduzir estas mulheres à obesidade;

- para homens de 10 a 17 anos e acima de 
60 anos, bem como para mulheres de 11 a 17 anos, conduziriam à desnutrição simplesmente por não incluírem provisão para as atividades físicas ocupacionais;

- para homens entre 18 e 59 anos, só podem ser usados para calcular o RHE médio da população total, pois seu uso para os residentes em áreas rurais ou urbanas separadamente conduziria à desnutrição ou à obesidade, respectivamente.

Além disto, este artigo mostra que os requerimentos baseados nos valores estimados de NAF aproximam-se, em média, dos requerimentos calculados pelo método fatorial, para todas as estratificações utilizadas. Por fim, considerando que existe, no âmbito do Sistema Estatístico Nacional, informação suficiente para estimar valores de NAF mais adequados a diversas estratificações da população brasileira do que os recomendados, este artigo sugere o cálculo dos requerimentos com base nos valores de NAF estimados para o País.

Palavras-chave: Requerimentos energéticos. Pesquisa. Metabolismo de energia. Métodos de estimação.

\section{Abstract}

The international recommendations on human energy requirements (ER), proposed by FAO/WHO/UNU in 1985, represent a significant methodological progress since they changed the method of estimating ER from the average energy intake of healthy people to the daily energy expenditure of the population. This change was based on the observation that estimation based on energy intake yielded a circular argument in which the intake was not the most appropriate way to maintain healthy weight and optimal levels of physical activity for health on the long run.

The 1994 meeting of the International Dietary Energy Consultative Group, published in 1996, as well as papers by national and international authors indicate that the principal problems in the proposed method are related to:

- the adequacy of the equations to estimate the basal metabolic rate (BMR);

- the physical activity level (PAL) values;

- the mechanisms of adaptation;

- the method proposed to children under 10 years.

For Brazil, the BMR equations tend to overestimate adults' BMR and the PAL values are not appropriated to the population, as shown by recent studies on BMR measurement and PAL values estimation.

Comparing the ER assessed by the factorial method (based on the duration of the activities and the correspondent integrated energy indexes) with the simplified one (based on recommended PAL values), this paper shows that the recommended PAL values:

- for 18-59 year-old women represent higher ER (from $11 \%$ to $17 \%$ ) than necessary which could make these women become obese;

- for 10-17 and 60+ year-old males and 1117 year-old females will lead to undernutrition by not incorporating provisions for the occupational activities;

- for 18-59 year-old males can only be used to calculate the mean for the total popu- 
lation, because its use for the rural and urban populations separately will lead to under and overnutrition, respectively.

At last, the paper shows that the mean ER based on the estimated PAL values is very near to the mean ER based on the factorial method for all the strata defined. Considering that the Brazilian Official Statistical System has enough information to estimate PAL values more adequate to several stratifications of the population, this paper suggests determining ER based on the national estimated PAL values.

Keywords: Energy requirements. Surveys. Energy metabolism. Estimation methods.

\section{Introdução}

A partir de 1985, especialistas da Organização das Nações Unidas para Agricultura e Alimentação, da Organização Mundial da Saúde e da Universidade das Nações Unidas $^{1}$ decidiram modificar o método de determinação dos requerimentos de energia, que até então era usado a partir de coeficientes (kcal/kg) estabelecidos pela observação da ingestão de pessoas saudáveis, para baseá-lo na estimação dos componentes do gasto energético (GE) total diário. Esta modificação decorreu da constatação de que a determinação dos requerimentos com base em ingestões observadas era um argumento circular, uma vez que não eram elas, forçosamente, que mantinham a massa corporal (MC) desejável e os níveis ótimos de atividade física para a saúde a longo prazo.

Apesar desse significativo aprimoramento no método, as Recomendações Internacionais para Requerimentos Humanos de Energia de $1985^{1}$ têm ainda fontes de inadequação decorrentes da estimação da taxa metabólica basal, dos valores de nível de atividade física recomendados (NAFR) e do método de cálculo dos requerimentos para menores de 10 anos, além dos problemas advindos dos mecanismos de adaptação.

Este trabalho discute as referidas recomendações de 1985 e, a partir da comparação de duas formas propostas para o cálculo dos requerimentos energéticos, realizada com os dados de uma pesquisa brasileira sobre consumo alimentar e orçamentos familiares, mostra que os NAFR não são adequados a estratos da população brasileira, apesar de, na média nacional, as diferenças não serem grandes.

\section{As recomendações internacionais de 1985}

Nas recomendações internacionais para os requerimentos humanos de energia ${ }^{1}$, os especialistas enfatizaram três aspectos:

- a manutenção da saúde a longo prazo, acrescentando aos requerimentos ener- 
géticos uma provisão para a prática de atividades físicas que objetivam manter a aptidão física do ponto de vista muscular e cardiovascular;

- a necessidade de incluir uma provisão para a atividade física socialmente desejável, além da provisão para a atividade economicamente necessária, que já constava das recomendações anteriores ${ }^{2}$;

- que o cálculo dos requerimentos energéticos deveria ser baseado nos componentes de gasto energético (GE) e expresso como múltiplo da taxa metabólica basal (TMB).

Para as crianças até 9 anos, por falta de dados confiáveis sobre a TMB, as recomendações de 1985 mantiveram o método usado até então, propondo coeficientes de kcal/ kg que, multiplicados pela MC.

Para determinar os requerimentos energéticos das pessoas de 10 anos completos ou mais, James e Schofield ${ }^{3}$ e o relatório de $1985^{1}$ sugerem duas aproximações:

- levantamento das atividades em períodos do dia e uso do índice energético integrado (IEI), calculado como a razão entre o dispêndio energético de uma atividade (tarefa), em um certo período de tempo, e o dispêndio do metabolismo basal, no mesmo período de tempo;

- valores únicos para o dia em função do nível de atividade física ocupacional (NAFO) e uso dos valores de nível de atividade física (NAF), definidos como a razão entre a energia total gasta no dia e a TMB, ou seja, uma extensão do IEI quando são consideradas todas as atividades de um dia.

A primeira aproximação, também conhecida como método fatorial, consiste em desagregar o dia em função dos grupos de atividades similares e sua duração, o que normalmente fornece requerimentos individuais mais acurados do que a segunda aproximação, que utiliza os valores de NAF.

James e Schofield ${ }^{3}$ sintetizaram os valores de NAF recomendados (NAFR) pelo relatório de $1985^{1}$ para a atividade física desejável de adolescentes e adultos, levando em conta médias populacionais, e de adultos, considerando o NAFO, no qual é estimada uma duração máxima de oito horas de trabalho diário. Estes valores recomendados foram determinados a partir de cálculo teórico de levantamento fatorial do custo energético e da duração das atividades diárias e ocupacionais, classificadas como leve, moderada e pesada, ou seja, os valores de NAFR não foram baseados em medidas efetivamente realizadas.

Nos anos 90, o Grupo Consultivo Internacional de Energia da Dieta/International Dietary Energy Consultative Group (IDECG), recomendou que fossem mantidos os princípios gerais de cálculo dos requerimentos de energia e sua definição, contidos no relatório de $1985^{1}$, e que fossem produzidos mais dados de custo energético de diferentes atividades, para cálculo de IEI e NAF, em todos os grupos etários ${ }^{4-6}$.

O IDECG ${ }^{7}$ identificou, particularmente com relação aos menores de 18 anos completos, que:

- as recomendações superestimam os requerimentos para crianças abaixo de oito anos completos;

- as recomendações subestimam, principalmente em áreas rurais de países em desenvolvimento, os requerimentos a partir dos 12 anos;

- não há garantias para recomendar, para crianças abaixo de 10 anos, o acréscimo de $5 \%$ dos requerimentos para permitir um nível de atividade física socialmente desejável.

A partir do reconhecimento de que há grande diferenciação no estilo de vida e no nível de atividade física de adolescentes e crianças acima de 5 anos entre os países desenvolvidos e em desenvolvimento, o IDECG $^{7}$ sugere que as recomendações para esse grupo sejam baseadas em três níveis de atividade física habitual, como é feito para os adultos (18 anos ou mais) no relatório de $1985^{1}$.

Com relação aos adultos, apesar de o IDECG recomendar a manutenção dos valores de $\mathrm{NAFR}^{5,8}$, vários autores têm sugerido uma reavaliação, já que os dados de GE obtidos pela técnica da água duplamente 
marcada $(\mathrm{ADM})$ tendem a ser maiores do que os valores estabelecidos nas recomendações $^{9,10}$. De qualquer forma, todos concordam que é necessário obter mais dados para melhor determinar esses valores ${ }^{5,11}$.

Particularmente com relação a populações de países em desenvolvimento, os dados de GE são mais escassos e, como existe dificuldade de se realizar estudos com ADM nesses países, há necessidade de se estimar valores de NAF que possam ser mais apropriados para essas populações e seus vários segmentos.

Na reunião de especialistas para a elaboração das novas recomendações sobre requerimentos de energia, realizada em outubro de 2001, na sede da FAO, apesar de o método de ADM ter sido considerado como o padrão ouro para qualquer comparação, os princípios gerais das recomendações de 1985, baseados na TMB, IEI e NAF, foram mantidos. Diante do seu custo, a ADM deverá ser o método referendado para grupos específicos, como bebês, para os quais princípios de 1985 não deram bom resultado. Neste sentido, estudos para medição da TMB e estimação de valores de NAF manterão sua importância no contexto do cálculo dos requerimentos, principalmente nos países em desenvolvimento.

Dados brasileiros ${ }^{12,13}$ têm indicado que as equações de predição da FAO conduzem a valores médios de TMB de 12,5\% a 13,5\% maiores do que aqueles obtidos por calorimetria indireta. De fato, em revisão recente, Wahrlich e Anjos ${ }^{14}$ registraram que em 10 estudos, dentre 16 encontrados na literatura, a calorimetria indireta indicou que as equações de predição da FAO superestimavam a TMB e que esta superestimação era maior entre as mulheres do que entre os homens. Em parte, o problema das equações da FAO decorre da amostra de pessoas cujas medições da TMB foram utilizadas em sua determinação, visto que $50 \%$ dos indivíduos eram italianos ${ }^{14}$.

No entanto, não existe grande disponibilidade de calorímetros indiretos e a TMB acaba sendo estimada por meio de equações, tanto em pesquisas populacionais quan- to na clínica médica. Neste sentido, a escolha do valor de MC usado nas equações para estimar a TMB passa a ter um papel importante, na medida em que cada escolha representa um conceito distinto de requerimento energético e decorre de um objetivo específico. Dentre as alternativas de valor de MC destacam-se:

- a MC observada;

- a MC de referência;

- a MC saudável ${ }^{15}$.

Vasconcellos e Anjos ${ }^{16}$ indicam que o uso dos valores da MC observada é adequado aos estudos de seguridade e abastecimento alimentar de uma população, quando o objetivo é a determinação da demanda de energia proveniente dos alimentos. Os valores de referência são usados quando o objetivo é determinar requerimentos que assegurem, a todos, tamanho e composição corporais compatíveis com a boa saúde a longo prazo e um nível de atividade física socialmente desejável e economicamente necessário. No entanto, estes valores pressupõem a existência de dados antropométricos de uma população de referência, por definição sadia. Por fim, os valores de MC saudável servem para estabelecer diretrizes dietéticas para uma população, determinando requerimentos energéticos que servem principalmente para prevenir a obesidade e assegurar um mínimo de reservas energéticas para o organismo. A MC Saudável, no entanto, foi definida apenas para adultos de 21 anos completos ou mais, pois os especialistas envolvi$\operatorname{dos}^{15}$ consideraram que sua definição para menores de 21 anos requeria mais estudos para poder lidar com o crescimento e o desenvolvimento de crianças e adolescentes.

Ao fazer a escolha do valor de MC não se deve esquecer que os requerimentos energéticos de uma população, definidos em termos biológicos (valores de referência da MC), correspondem a uma redistribuição de energia entre os membros dessa população, quando comparado ao conceito de demanda por energia proveniente dos alimentos (valores observados de MC), na medida em que os requerimentos energéticos são reduzidos para os que têm valor de MC acima do valor 
de referência e aumentados para os que têm valor de MC inferior ao de referência.

As recomendações de 1985 estabelecem requerimentos em termos biológicos e sugerem o uso dos valores de MC de referência baseados em dados disponíveis da população de referência do país ou de uma subpopulação supostamente sadia e, caso não existam dados locais, sugerem o uso de dados internacionais ${ }^{3}$.

\section{Material e métodos}

Os dados, utilizados neste artigo, derivam do Estudo Nacional da Despesa Familiar (ENDEF), uma pesquisa de consumo alimentar e orçamentos familiares, realizada pela Fundação Instituto Brasileiro de Geografia e Estatística (IBGE), com coleta de dados realizada entre agosto de 1974 e agosto de $1975^{17,18}$. A informação do ENDEF baseia-se nos dados de 53.311 famílias e 267.446 pessoas, de uma amostra probabilística de domicílios selecionada em quatro estágios (municípios, setores do Censo Demográfico 1970, subsetores e domicílios), cujo tamanho foi definido para produzir estimativas representativas da população brasileira de 22 áreas geográficas do País ${ }^{19}$, exceto as áreas rurais das macrorregiões Norte e Centro-Oeste.

O método de pesquisa adotado consistiu na realização, por sete dias consecutivos, de tantas entrevistas diárias quantas fossem as refeições principais realizadas pela família no dia, para pesar os alimentos que seriam consumidos na refeição seguinte, bem como os resíduos não-alimentares e desperdícios (entendidos como as quantidades de alimentos pesadas para consumo e que não foram ingeridas durante a semana de pesquisa) da refeição anterior. Além da pesagem de alimentos, foram registradas as presenças dos comensais (moradores ou não no domicílio selecionado) às refeições diárias, dados pessoais (sexo, idade, massa corporal, estatura, ocupações exercidas na semana de entrevista, horas trabalhadas em cada ocupação, rendimento) e os dados familiares de despesas e receitas, necessários para a elaboração do orçamento familiar ${ }^{20}$. As despesas incluíram, além dos fluxos monetários (compras), os fluxos não-monetários associados à produção própria, às trocas, às doações recebidas, aos pagamentos em bens por serviços prestados pelos membros da família e aos bens retirados do estoque de negócio gerido por um membro da família. A despesa per capita foi obtida dividindo-se a despesa familiar pelo número de membros da família ${ }^{20}$.

Das variáveis do ENDEF que fornecem os requerimentos energéticos diários das pessoas, duas são de particular interesse para este trabalho: requerimento energético com o método fatorial e requerimento energético com NAF recomendado (NAFR). Para as pessoas de 10 anos ou mais, estas duas variáveis foram calculadas tendo por base as estimativas de TMB obtidas a partir das equações de predição recomendadas para uso internacional $^{1}$ com valores de massa corporal (MC) de referência, por sexo, idade e estatura, que foram estimados com base em modelos de regressão linear aplicados sobre os dados de uma subamostra de pessoas supostamente sadias, de acordo com critérios baseados na despesa per capita da família ${ }^{21}$.

Para a primeira variável de requerimento energético, foi aplicado o método fatorial descrito no Quadro 1, considerando-se: oito horas de sono; duas horas de atividades socialmente desejáveis; 20 minutos de exercício cardiovascular, se a pessoa não era ocupada ou se sua ocupação era de NAFO leve; um sétimo das horas trabalhadas por semana com um IEI correspondente ao NAFO de cada ocupação exercida na semana de pesquisa; e as demais horas do dia para atividades com gasto energético de manutenção. Para definir o NAFO associado a cada ocupação foi utilizado o trabalho de Ribeiro ${ }^{22}$, que, baseando-se na descrição contida na Classificação Brasileira das Ocupações ${ }^{23}$ e seguindo aproximadamente a distribuição percentual do tempo de atividades específicas presentes nas recomendações de $1985^{1}$ associada a outras fontes, classificou as ocupações observadas na Pesquisa Nacional de Saúde e Nutrição - PNSN ${ }^{24}$ em NAFO leve, moderado e pesado. Esta categorização, quando comparada ao estado nutricional de 
adultos, avaliado por meio das medidas antropométricas, mostrou aderência ${ }^{25,26}$.

Para a segunda variável, requerimento energético com NAF recomendado, o valor da TMB foi multiplicado pelo valor de NAFR $\left(=\mathrm{GE}_{24 \mathrm{~h}} / \mathrm{TMB}_{24 \mathrm{~h}}\right)$. James e Schofield ${ }^{3}$ sintetizaram os valores de NAFR, propostos no relatório de $1985^{1}$, para:

- a atividade física desejável de adolescentes (10 a 17 anos) e adultos (18 anos ou mais), considerando médias populacionais por sexo, grupo etário e grau de desenvolvimento do país, que devem ser usados quando não se dispõe de informação sobre o NAFO (Tabela 1.8 de James e Schofield ${ }^{3}$ );

- adultos, considerando o NAFO, no qual é pressuposta uma duração máxima de oito horas de trabalho diário (Tabela 3.5 de James e Schofield ${ }^{3}$ ) e não fica claro o valor de NAFR a ser associado aos adultos não-ocupados.

Na variável utilizada neste trabalho, foram usados os seguintes valores: (1) para os não-ocupados de 10 a 17 anos, o valor de NAFR para o sexo e idade; (2) para os ocupados de 10 a 17 anos, o maior valor entre o NAFR para o sexo e idade e o NAFR para o sexo e o NAFO associado à ocupação de maior número de horas trabalhadas na semana de pesquisa; (3) para os não-ocupados de 18 anos ou mais, o valor de NAFR para o sexo e o NAFO leve; e (4) para os ocupados de 18 anos ou mais, o valor de NAFR para o sexo e o NAFO relativo à ocupação de maior número de horas trabalhadas na semana de pesquisa.

Em termos ideais, o requerimento de uma pessoa não deveria mudar em decorrência do método usado em sua determina-

Quadro 1 - Duração e recomendação energética por sexo, segundo o nível de atividade física ocupacional e as atividades ${ }^{1}$

Chart 1 - Energy requirements and duration for each gender, according to the level of occupational physical activity and activities ${ }^{1}$

\begin{tabular}{|c|c|c|c|c|}
\hline \multirow[b]{2}{*}{$\begin{array}{l}\text { Nível de atividade física ocupacional } \\
\text { (NAFO) e atividades }\end{array}$} & \multicolumn{2}{|c|}{ Homens } & \multicolumn{2}{|c|}{ Mulheres } \\
\hline & $\begin{array}{l}\text { Duração } \\
\text { (horas) }^{2}\end{array}$ & $\begin{array}{c}\text { Gasto } \\
\text { energético }\end{array}$ & $\begin{array}{l}\text { Duração } \\
\text { (horas) }^{2}\end{array}$ & $\begin{array}{c}\text { Gasto } \\
\text { energético }\end{array}$ \\
\hline \multicolumn{5}{|l|}{ Pessoas com NAFO leve } \\
\hline Sono & 8 & $1,0 \times \mathrm{TMBh}$ & 8 & $1,0 \times T M B h$ \\
\hline Atividades socialmente desejáveis & 2 & $3,0 \times \mathrm{TMBh}$ & 2 & $3,0 \times \mathrm{TMBh}$ \\
\hline Exercício cardiovascular & $1 / 3$ & $6,0 \times T M B h$ & $1 / 3$ & $6,0 \times \mathrm{TMBh}$ \\
\hline Trabalho & HTS $\div 7$ & $1,7 \times \mathrm{TMBh}$ & HTS $\div 7$ & $1,7 \times \mathrm{TMBh}$ \\
\hline Outras atividades & $14-\mathrm{HTS} \div 7-1 / 3$ & $1,4 \times \mathrm{TMBh}$ & $14-\mathrm{HTS} \div 7-1 / 3$ & $1,4 \times \mathrm{TMBh}$ \\
\hline Total do dia & 24 & 24 & & \\
\hline \multicolumn{5}{|l|}{ Pessoas com NAFO moderado } \\
\hline Sono & 8 & $1,0 \times \mathrm{TMBh}$ & 8 & $1,0 \times \mathrm{TMBh}$ \\
\hline Atividades socialmente desejáveis & 2 & $3,0 \times \mathrm{TMBh}$ & 2 & $3,0 \times T M B h$ \\
\hline Trabalho & HTS $\div 7$ & $2,7 \times T M B h$ & HTS $\div 7$ & $2,2 \times \mathrm{TMBh}$ \\
\hline Outras atividades & $14-\mathrm{HTS} \div 7$ & $1,4 \times \mathrm{TMBh}$ & $14-\mathrm{HTS} \div 7$ & $1,4 \times \mathrm{TMBh}$ \\
\hline Total do dia & 24 & 24 & & \\
\hline \multicolumn{5}{|l|}{ Pessoas com NAFO pesado } \\
\hline Sono & 8 & $1,0 \times T M B h$ & 8 & $1,0 \times \mathrm{TMBh}$ \\
\hline Atividades socialmente desejáveis & 2 & $3,0 \times T M B h$ & 2 & $3,0 \times \mathrm{TMBh}$ \\
\hline Trabalho & HTS $\div 7$ & $3,8 \times \mathrm{TMBh}$ & HTS $\div 7$ & $2,8 \times \mathrm{TMBh}$ \\
\hline Outras atividades & $14-\mathrm{HTS} \div 7$ & $1,4 \times \mathrm{TMBh}$ & $14-\mathrm{HTS} \div 7$ & $1,4 \times \mathrm{TMBh}$ \\
\hline Total do dia & 24 & 24 & & \\
\hline
\end{tabular}

${ }^{1}$ Adaptado de FAO/WHO/UNU:Tabelas 8 a 14 / ' Adapted from FAO/WHO/UNU. Tables 8 to 14.

${ }^{2} \mathrm{HTS}$ é o total de horas trabalhadas na semana nas ocupações que estavam sendo exercidas na semana de pesquisa, que, dividida por sete, indica o número médio de horas trabalhadas por dia / ${ }^{2}$ HTS corresponds to total hours worked during the week in the occupations that were being performed during the week of the survey. Divided by seven, it indicates the mean number of hours worked per day.

${ }^{3}$ Ético corresponde ao produto do índice energético integrado - IEI - pela taxa metabólica basal para uma hora (TMBh) / ${ }^{3}$ Ético corresponds to the product of the integrated energy rate - IEI - by the basal metabolic rate for one hour (TMBh). 
ção e, portanto, deveria ser usado um valor de NAF que tornaria iguais os valores das duas variáveis de requerimento energético referidas. Considerando que o valor do requerimento energético com o método fatorial é mais acurado que o outro, sua divisão pela TMB forneceria um valor de NAF que reflete melhor a atividade física da pessoa do que o valor recomendado de NAF (que foi concebido para representar a atividade física de grupos populacionais). Os valores individuais de NAF, obtidos pela divisão referida, podem ser usados para determinar os valores de NAF estimados (NAFE) para a população ou para diferentes estratos da população.

De fato, Vasconcellos ${ }^{21}$ mostrou que os valores de NAFE independem da TMB e podem ser estimados a partir de dados sobre as atividades (que definem os IEIs a serem aplicados) e suas durações, ambos obtidos em uma amostra representativa da população, pois o valor de NAF de cada pessoa corresponde a uma média dos IEIs de suas atividades ponderados pelas durações das atividades. Se as atividades consideradas se limitarem à ocupação exercida e às atividades recomendadas, como indicado no Quadro 1, existe, no âmbito do Sistema Estatístico Nacional, criado pela Lei Federal no 5878 , de 11/05/73, do qual a Fundação Instituto Brasileiro de Geografia e Estatística (IBGE) é o coordenador, informação disponível suficiente para determinar os valores de NAFE usando-se o método de estimação formalizado por Vasconcellos e Anjos ${ }^{27}$.

Para este estudo, os valores de NAFE foram obtidos para duas estratificações distintas. A primeira estratificação levou em conta sexo, classe de idade (ano a ano de 10 a 19 anos; grupos qüinqüenais de 20 a 79 anos; e 80 anos ou mais) e os 22 estratos geográficos da amostra do ENDEF (apesar de os resultados serem apresentados de forma agregada para o total nacional; regiões metropolitanas; áreas urbanas e áreas rurais), tendo sido concebida para aplicação em pesquisas de abrangência nacional que não coletam informação sobre a condição ocupacional das pessoas e, portanto, os va- lores de NAFE devem refletir as características ocupacionais da população alvo da avaliação.

Na segunda estratificação, pensada para aplicação em pesquisas de orçamentos familiares, incluiu-se uma proxyde renda. Esta estratificação baseia-se no sexo, na idade (ano a ano de 10 a 17 anos; 18 e 19 anos; 20 a 24 anos; 25 a 29 anos; grupos decenais de 30 a 69 anos; e 70 anos ou mais), nos 22 estratos da amostra do ENDEF e nas classes de despesa delimitadas pelos quartis da distribuição da despesa per capita.

Os valores de NAFE, obtidos pelo método descrito, foram comparados com os de NAFR, tendo sido também utilizados para o cálculo do requerimento energético com NAF estimado. Este requerimento, calculado da mesma forma descrita para o requerimento com NAF recomendado, foi comparado aos outros dois requerimentos definidos.

\section{Resultados e Discussão}

Na Tabela 1, estão apresentados os valores de NAF da primeira estratificação, calculados segundo sexo, classe de idade e os 22 estratos geográficos. Para cada sexo, foram incluídas na Tabela as colunas de NAF recomendado e de NAF estimado para o total das áreas abrangidas pelo ENDEF.

Pode-se observar que as principais diferenças entre os valores recomendados e os estimados ocorrem:

- para homens entre 10 e 17 anos e mulheres entre 11 e 17 anos, devido ao fato de as recomendações de $1985^{1,3}$ incluírem apenas as provisões necessárias ao crescimento e às atividades socialmente desejáveis, ignorando o gasto energético para as atividades ocupacionais, que foram considerados nos valores estimados; - para os homens de 60 anos ou mais, devido ao fato de as recomendações de $1985^{1,3}$ proporem os valores de NAFO apenas para adultos de 18 a 59 anos e os valores estimados levarem em conta a informação de ocupação;

- para as mulheres a partir de 18 anos de idade, porque o gasto energético relati- 
Tabela 1 - Valores de nível de atividade física (NAF) recomendados e estimados por sexo, segundo a classe de idade

Table 1 - Recommended and estimated values for the level of physical activity (NAF) per gender, according to age bracket

\begin{tabular}{lcccc}
\hline Classe de idade & \multicolumn{2}{c}{ Homens } & \multicolumn{2}{c}{ Mulheres } \\
& $\begin{array}{c}\text { Valores de NAF } \\
\text { recomendados }{ }^{1}\end{array}$ & $\begin{array}{c}\text { Valores de NAF } \\
\text { estimados }\end{array}$ & $\begin{array}{c}\text { Valores de NAF } \\
\text { recomendados }{ }^{1}\end{array}$ & $\begin{array}{c}\text { Valores de NAF } \\
\text { estimados }\end{array}$ \\
\hline 10 anos & 1,76 & 1,78 & 1,65 & 1,65 \\
11 anos & 1,72 & 1,75 & 1,62 & 1,63 \\
12 anos & 1,69 & 1,74 & 1,60 & 1,61 \\
13 anos & 1,67 & 1,75 & 1,58 & 1,60 \\
14 anos & 1,65 & 1,76 & 1,57 & 1,60 \\
15 anos & 1,62 & 1,79 & 1,54 & 1,58 \\
16 anos & 1,60 & 1,80 & 1,52 & 1,58 \\
17 anos & 1,60 & 1,82 & 1,52 & 1,58 \\
18 anos & 1,82 & 1,79 & 1,67 & 1,54 \\
19 anos & 1,82 & 1,78 & 1,67 & 1,54 \\
20 a 24 anos & 1,82 & 1,81 & 1,67 & 1,53 \\
25 a 29 anos & 1,82 & 1,82 & 1,67 & 1,52 \\
30 a 34 anos & 1,82 & 1,83 & 1,67 & 1,52 \\
35 a 39 anos & 1,82 & 1,83 & 1,67 & 1,53 \\
40 a 44 anos & 1,82 & 1,82 & 1,67 & 1,53 \\
45 a 49 anos & 1,82 & 1,82 & 1,67 & 1,52 \\
50 a 54 anos & 1,82 & 1,79 & 1,67 & 1,52 \\
55 a 59 anos & 1,82 & 1,76 & 1,67 & 1,51 \\
60 a 64 anos & 1,51 & 1,72 & 1,56 & 1,49 \\
65 a 69 anos & 1,51 & 1,66 & 1,56 & 1,49 \\
70 a 74 anos & 1,51 & 1,60 & 1,56 & 1,48 \\
75 a 79 anos & 1,51 & 1,56 & 1,56 & 1,47 \\
80 anos ou mais & 1,51 & 1,52 & 1,56 & 1,46 \\
\hline
\end{tabular}

${ }^{1}$ Valores de nível de atividade física (NAF) recomendados pelo relatório FAO/WHO/UNU de 1985.

1 Values for level of physical activity recommended by the $1985 \mathrm{FAO} / \mathrm{WHO} / \mathrm{UNU}$ report.

vo ao trabalho doméstico não pôde ser considerado na estimação dos valores de NAF (o ENDEF não levantou os dados necessários) e a proporção de mulheres adultas economicamente ocupadas em 1974/75 era, muito provavelmente, inferior à proporção usada na determinação dos valores recomendados.

De fato, entre as mulheres de 10 anos ou mais da população brasileira de 1975, apenas, $33,2 \%$ eram ocupadas, enquanto entre os homens de 10 anos ou mais a porcentagem de ocupados era de $66,9 \%{ }^{21}$.

A Figura 1 desagrega os dados da Tabela 1 para os conjuntos de áreas metropolitanas, urbanas e rurais pesquisadas pelo ENDEF, ressaltando as diferenças entre os valores de NAFR e NAFE.
O exame das curvas dos valores de NAFR e NAFE por área geográfica (Figura 1) mostra que os valores estimados para o estrato rural são sistematicamente bem superiores àqueles dos estratos metropolitanos e urbanos, indicando que:

- as ocupações agropecuárias, mais freqüentes no estrato rural, por seu esforço físico e sua duração, têm um gasto energético, em média, bem maior do que as ocupações urbanas (geralmente dos setores secundário e terciário da economia);

- os valores estimados para o estrato rural influíram de forma importante nos valores médios nacionais, o que significa que a variação da proporção de população ocupada rural, ao longo do tempo, altera os valores de NAFE para o total do País. 

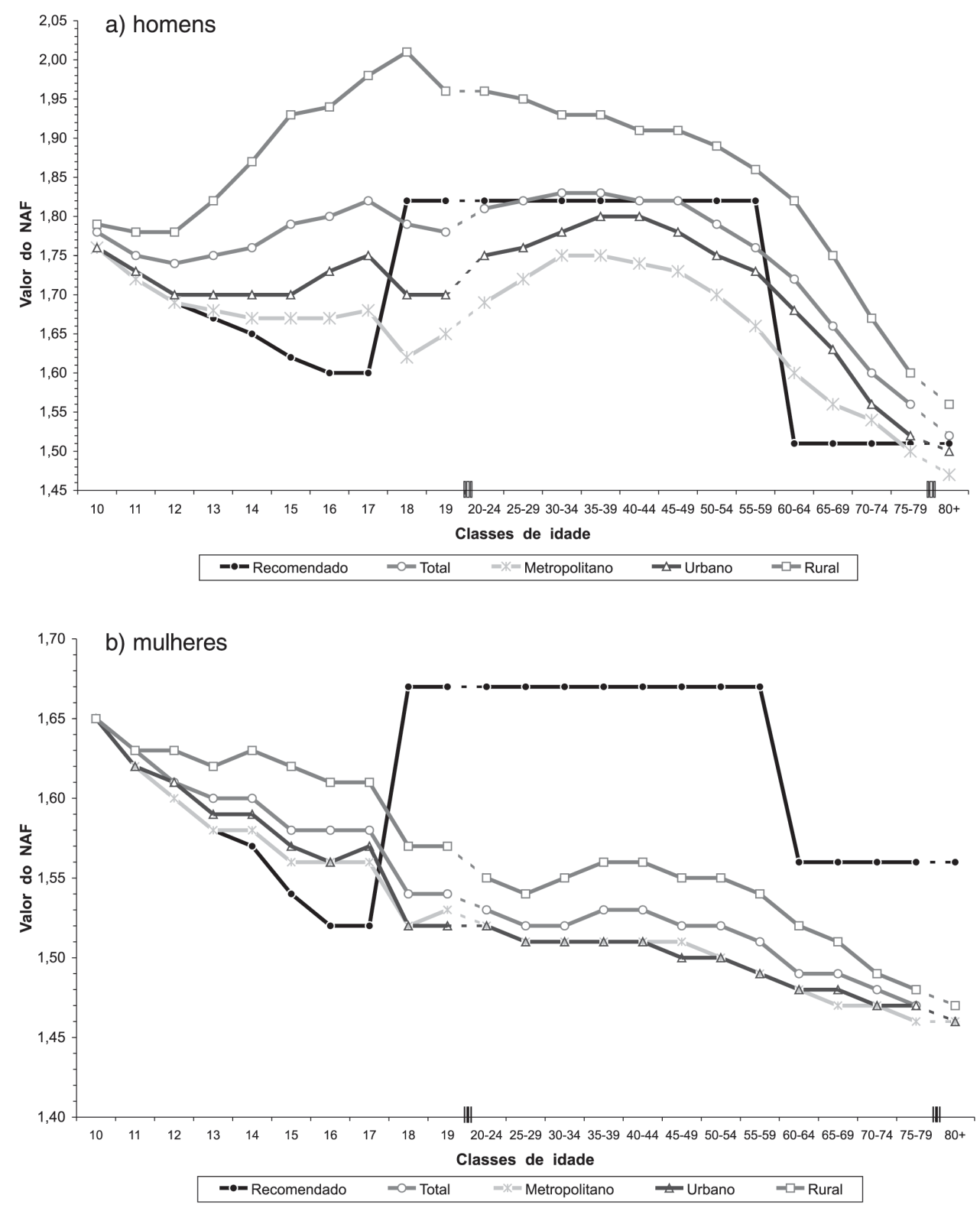

Figura 1 - Valores de NAF estimados e recomendados, por sexo e classe de idade. Figure 1 - Estimated and recommended NAF values per gender and age bracket.

Além disso, é provável que as diferenças entre os estratos rurais e urbanos possam ser ainda maiores, já que não se levou em consideração o nível de desenvolvimento tecnológico do trabalho em cada estrato. Fica evidente, portanto, que os valores de NAF deveriam ser, pelo menos, regionalizados, já que as características demográficas e ocupacionais são bastante distintas, particularmente para os homens.
Em síntese, os resultados apresentados na Tabela 1 e na Figura 1 significam que os valores de NAFR:

- para mulheres a partir de 18 anos determinariam valores de requerimento energético superiores às suas necessidades, com acréscimos à TMB variando entre 11 e $17 \%$ acima do necessário para a média do grupo, o que fatalmente conduziria estas mulheres à obesidade; 
- para homens entre 18 e 59 anos só podem ser utilizados para a média nacional, pois sua aplicação a qualquer estrato isolado implica a desnutrição no estrato rural e a obesidade nos demais estratos; e

- para homens entre 10 e 17 anos e acima de 60 anos, bem como para mulheres entre 12 e 17 anos, conduziriam à desnutrição por não incluírem provisão para as atividades físicas ocupacionais.

O segundo grupo de valores estimados de NAF foi obtido com a estratificação por sexo, idade, estrato da amostra e quartil da despesa corrente per capita, gerando 2.816 ( 2 sexos $\times 16$ classes de idade $\times 22$ estratos $\times$ 4 classes de despesa) valores de NAF que foram utilizados para calcular o requerimento energético diário com NAF estimado, cuja comparação com os requerimentos energéticos diários com NAF recomendado e com método fatorial consta da Tabela 2 .

Observa-se, para a linha de total da Tabela 2, que o requerimento calculado com os valores de NAFR tem a maior média populacional. Esta tendência de superestimação dos requerimentos com NAFR, quando comparado ao calculado com o método fatorial, é conseqüência da super- estimação dos requerimentos entre as mulheres (69,9 kcal, em média), já que entre os homens o requerimento com NAFR subestima o requerimento médio populacional em $14,2 \mathrm{kcal}$. Estes fatos já haviam sido observados para os requerimentos baseados nas recomendações internacionais anteriores ${ }^{2} \mathrm{e}$ se deve à alocação constante de oito horas diárias de trabalho, que é um valor médio baixo para homens e muito alto para mulheres ${ }^{21}$.

A Tabela 2 indica que o requerimento com NAF estimado, em decorrência do método de estimação dos valores de NAFE, tem uma média muito próxima da média do requerimento com método fatorial, apresentando diferenças de 1,5 kcal para o total, 0,9 kcal entre os homens e 2,2 kcal entre as mulheres. Nesta Tabela observa-se também que a variabilidade do requerimento com método fatorial, medida pelo coeficiente de variação amostral, é maior do que a dos requerimentos com NAFR e com NAFE estimado, na medida em que no cálculo destes dois últimos perde-se a fonte de variação individual relacionada ao NAFO e ao número de horas trabalhadas.

Em resumo, os resultados da Tabela 2 mostram que o uso dos valores de NAFE é mais aconselhável do que o dos valores de

Tabela 2 - Características das distribuições dos requerimentos energéticos diários dos maiores de nove anos, segundo a forma de cálculo

Table 2 - Characteristics of distributions of daily energy requirements of individuals over nine years of age, according to the formula

\begin{tabular}{lcccc}
\hline $\begin{array}{l}\text { Forma de cálculo do } \\
\text { requerimento diário } \\
\text { de energia }\end{array}$ & $\begin{array}{c}\text { Média } \\
\text { populacional } \\
(\mathrm{kcal})\end{array}$ & $\begin{array}{c}\text { Doeficiente de } \\
\text { variação }(\%)\end{array}$ & $\begin{array}{c}\text { Mínimo } \\
(\mathrm{kcal})\end{array}$ & $\begin{array}{c}\text { Máximo } \\
(\mathrm{kcal})\end{array}$ \\
\hline $\begin{array}{l}\text { Total } \\
\quad \text { Com o método fatorial }\end{array}$ & 2320,3 & 22,7 & 1303 & 5078 \\
$\quad$ Com NAF estimado & 2318,8 & 19,8 & 1379 & 4186 \\
$\quad$ Com NAF recomendado & 2349,4 & 19,6 & 1388 & 4166 \\
\hline $\begin{array}{l}\text { Homens } \\
\text { Com o método fatorial }\end{array}$ & 2703,6 & 19,2 & 1357 & 5078 \\
$\quad$ Com NAF estimado & 2702,7 & 13,9 & 1519 & 4186 \\
$\quad$ Com NAF recomendado & 2689,4 & 16,4 & 1400 & 4166 \\
\hline Mulheres & & & & \\
$\quad$ Com o método fatorial & 1958,6 & 9,4 & 1303 & 3216 \\
$\quad$ Com NAF estimado & 1956,4 & 7,6 & 1379 & 2698 \\
$\quad$ Com NAF recomendado & 2028,5 & 7,9 & 1388 & 2939 \\
\hline
\end{tabular}


NAFR, uma vez que os valores de NAFE geram requerimentos médios mais próximos daqueles obtidos pelo método fatorial.

Por fim, para avaliar o comportamento do gasto energético ocupacional em relação ao nível de renda foi incluída a Figura 2, que apresenta as diferenças entre os valores de NAFE e NAFR para homens por classe de despesa corrente per capita.

Na Figura 2, observa-se, para todas as classes de despesa, que os valores recomendados para homens de 10 a 17 anos e de 60 anos ou mais são menores (diferenças positivas) do que os estimados. Além disto, com exceção de algumas classes de idade (provavelmente em decorrência do número pequeno de pessoas ocupadas na amostra por classe de despesa), observa-se uma clara tendência a quanto maior a classe de despesa menor o valor do NAFE, indicando que o gasto ocupacional é inversamente relacionado ao nível de renda (ou despesa), pois são as pessoas mais pobres e menos instruídas que desempenham as ocupações de maior desgaste físico, enquanto os mais ri- cos e mais instruídos desempenham ocupações de menor desgaste físico. Este resultado significa que o uso dos valores de NAFR também são inadequados a estratos econômicos da população, na medida em que determinam requerimentos que fatalmente conduziriam a população à desnutrição ou à obesidade.

Em função dos resultados apresentados, pode-se concluir que as recomendações internacionais de 1985, apesar de representarem um importante avanço nos métodos de determinação dos requerimentos energéticos, ainda têm deficiências importantes.

As equações de predição recomendadas para uso internacional não foram suficientemente testadas para a população brasileira e há evidências de que superestimam a taxa metabólica basal.

Os valores de NAFE mostraram que os recomendados por sexo e idade para países menos desenvolvidos não são adequados a estratos da população brasileira, apesar de não implicarem um grande erro no requerimento médio nacional.

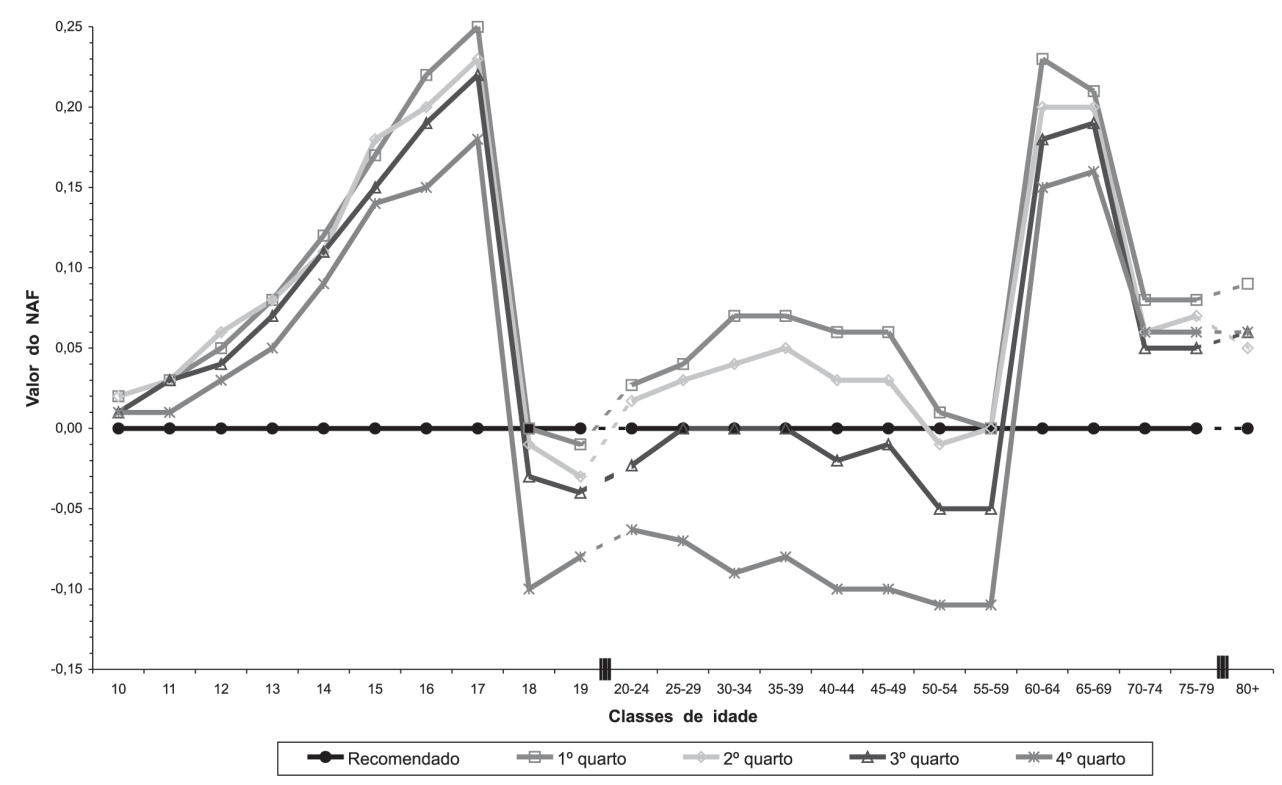

Figura 2 - Diferenças entre os valores de NAF estimados e os recomendados para homens, por classe de despesa corrente per capita e classe de idade.

Figure 2 -Differences between estimated and recommended NAF values for men, by class of current per capita expenses and age bracket. 
Pode-se argumentar que os dados apresentados derivam de uma pesquisa antiga e que o aumento do número de mulheres ocupadas, apontados pelos Censos Demográficos 1991 e 2000, indicam que a diferença entre o NAFR e o NAFE seria menor do que a apresentada e, portanto, que estes dados não constituem evidência suficiente para condenar os valores de NAFR. No entanto, os dados deste artigo mostram uma importante variação de gasto energético entre os diferentes segmentos da população brasileira (urbana e rural, por sexo, por nível de renda) e, portanto, que "valores internacionais" por sexo e idade não podem ser aplicados a cada segmento isolado da população. Em termos estatísticos, os resultados deste artigo indicam que a dispersão entre os valores médios de NAF de cada estrato (ou segmento) da população invalida a aplicação de um valor constante para estimar o gasto energético para um estrato isolado.

Muito mais importante do que qualquer estimativa apresentada é a discussão metodológica exemplificada por estes dados. E a pergunta que fica é se a dispersão entre as médias de gasto energético dos diferentes segmentos da população foi reduzida a ponto de permitir o uso de valores de NAF constantes por segmento? E a distribuição da população por ocupação, observada nos dois censos citados, não indica que a dispersão tenha sido reduzida a ponto de poder ser ignorada sem repercussões importantes.

Neste sentido, a aplicação das recomendações de 1985 para um indivíduo ou para estratos da população brasileira deve ser analisada com cuidado. Para evitar os problemas apontados, os requerimentos devem ser calculados com valores estimados de NAF para o grupo em estudo.

\section{Referências}

1. Food and Agriculture Organization of the United Nations/World Health Organization/ University of the United Nations. Energy and Protein Requirements: Report of a Joint FAO/WHO/UNU ad hoc Expert Consultation. Geneva: WHO; 1985. (WHO - Technical Report Series, 724).

2. Food and Agriculture Organization of the United Nations/World Health Organization. Energy and Protein Requirements. Report of a Joint FAO/WHO ad hoc Expert Consultation. Rome: FAO; 1973. (FAO Nutrition Meeting Report Series, 52).

3. James WPT, Schofield EC. Human Energy Requirements: a Manual for Planners and Nutritionists. New York: FAO and Oxford University Press; 1990.

4. Butte NF, Henry CJK, Torun B. Report of the working group on energy requirements of infants, children and adolescents. Eur J Clin Nutr 1996; 50(Suppl 1): S188-9.
5. Buyckx M, Dupont JL, Durnin JVGA, Ferro-Luzzi A et al. Report of the working group on general principles of assessing energy requirements. Eur J Clin Nutr 1996; 50(Suppl 1): S186-7.

6. Dupont JL, Durnin JVGA, Ferro-Luzzi A, Roberts SB et al. Report of the working group on energy requirements of older individuals. Eur J Clin Nutr 1996; 50(Suppl 1): S192.

7. International Dietary Energy Consultative Group. Energy and protein requirements: proceedings of an IDECG workshop held at the London School of Hygiene and Tropical Medicine, UK, 1994. Eur J Clin Nutr 1996; 50(Suppl 1): S1-S197.

8. Shetty PS, Henry CJK, Black AE, Prentice AM. Energy requirements of adults: an update on basal metabolic rates (BMRs) and physical activity levels (PALs). Eur J Clin Nutr 1996; 50(Suppl 1): S12-23. 
9. Durnin JVGA. Energy requirements: general principles. Eur J Clin Nutr 1996; 50(Suppl 1): S2-10.

10. Haggarty P, McNeill G, Manneh MK, Davidson L, Milne E, Duncan G, Ashton J. The influence of exercise on the energy requirements of adult males in the UK. $\mathrm{Br} J$ Nutr 1994; 72: 799-813.

11. Heini AF, Minghelli G, Diaz E, Prentice AM, Schutz Y. Free-living energy expenditure assessed by two different methods in rural Gambian men. Eur J Clin Nutr 1996; 50: 284-9.

12. Cruz CL, Silva AF, Anjos LA. A taxa metabólica basal é superestimada pelas equações preditivas em universitárias do Rio de Janeiro, Brasil. Arch Latinoam Nutr 1999; 49(3): 232-7.

13. Wahrlich V, Anjos LA. Validação de equações de predição da taxa metabólica basal em mulheres residentes em Porto Alegre, RS, Brasil. Rev Saúde Pública 2001; 35(1): 39-45.

14. Wahrlich V, Anjos LA. Aspectos históricos e metodológicos da medição e estimativa da taxa metabólica basal: uma revisão da literatura. Cad Saúde Pública 2001; 17(4): 801-17.

15. American Institute of Nutrition. Report of the American Institute of Nutrition Steering Committee on Health Weight. J Nutr 1994; 124: 2240-3.

16. Vasconcellos MTL, Anjos LA. Taxa de adequação (ingestão/requerimento) de energia como indicador do estado nutricional das famílias: uma análise crítica dos métodos aplicados em pesquisas de consumo de alimentos. Cad Saúde Pública 2001; 17(3): 581-93.

17. Fundação Instituto Brasileiro de Geografia e Estatística. Estudo Nacional da Despesa Familiar ENDEF: Dados Preliminares, Consumo Alimentar Antropometria. Rio de Janeiro: IBGE; 1977. (ENDEF, v.1, t.1, pt.1 a pt.4).

18. Fundação Instituto Brasileiro de Geografia e Estatística. Estudo Nacional da Despesa Familiar ENDEF: Dados Preliminares, Despesa das Famílias. Rio de Janeiro: IBGE; 1978. (ENDEF, v.1, t.2, pt.1 a pt.7).
19. Vasconcellos MTL. Metodologia do Estudo Nacional da Despesa Familiar: Objetivos Descrição e Metodologia Usada no ENDEF. Rio de Janeiro: IBGE; 1983. (ENDEF, v.3, t.4, pt.1).

20. Vasconcellos MTL. Metodologia do Estudo Nacional da Despesa Familiar: Núcleo do Banco de Informações ENDEF. Rio de Janeiro: IBGE; 1983. (ENDEF, v.3, t.4, pt.2).

21. Vasconcellos MTL. Análise crítica dos métodos de avaliação nutricional de populações, a partir de dados de consumo familiar de energia [tese de doutorado]. Rio de Janeiro: Escola Nacional de Saúde Pública, Fundação Oswaldo Cruz; 2001.

22. Ribeiro FSN. Da Identidade do Pesquisado à Identidade da Pesquisa: os Trabalhadores Brasileiros na Pesquisa Nacional sobre Saúde e Nutrição [dissertação de mestrado]. Rio de Janeiro: Escola Nacional de Saúde Pública, Fundação Oswaldo Cruz; 1994.

23. Ministério do Trabalho. Classificação Brasileira de Ocupações. Brasília: Sistema Nacional de Emprego, Ministério do Trabalho; 1982.

24. Instituto Nacional de Alimentação e Nutrição/ Fundação Instituto Brasileiro de Geografia e Estatística/Instituto de Planejamento Econômico e Social. Pesquisa Nacional sobre Saúde e Nutrição: Resultados Preliminares. Brasília: INAN; 1990.

25. Burlandy L, Anjos LA. Acesso a vale refeição e estado nutricional de adultos beneficiários do programa de alimentação do trabalhador no Nordeste e Sudeste do Brasil, 1997. Cad Saúde Pública 2001; 17(6): 1457-64.

26. Anjos LA. Relationship between body mass index (BMI) and socioeconomic characteristics of the Brazilian adult population. Am J Human Biol 1993; 5(1): 133-4.

27. Vasconcellos MTL, Anjos L.. A simplified method for assessing physical activity level values for a country or study population. Eur J Clin Nutr, (no prelo). 